\title{
openheart Impact of collateralisation to a concomitant chronic total occlusion in patients with ST-elevation myocardial infarction: a subanalysis of the EXPLORE randomised controlled trial
}

Ivo M van Dongen, ${ }^{1}$ Joëlle Elias, ${ }^{1} \mathrm{~K}$ Gert van Houwelingen, ${ }^{2}$

Pierfrancesco Agostoni, ${ }^{3}$ Bimmer E P M Claessen, ${ }^{1}$ Loes P Hoebers, ${ }^{1}$ Dagmar M Ouweneel, ${ }^{1}$ Esther M Scheunhage, ${ }^{1}$ Ronak Delewi, ${ }^{1}$ Jan J Piek, ${ }^{1}$ Truls Råmunddal, ${ }^{4}$ Peep Laanmets, ${ }^{5}$ Erlend Eriksen, ${ }^{6}$ Matthijs Bax, ${ }^{7}$ Maarten J Suttorp, ${ }^{3}$ René J van der Schaaf, ${ }^{8}$ Jan G P Tijssen, ${ }^{1}$ José P S Henriques, ${ }^{1}$ on behalf of the EXPLORE investigators

\section{- Additional material is published online only. To view please visit the journal online (http://dx.doi.org/10.1136/ openhrt-2018-000810).}

To cite: van Dongen IM, Elias J, van Houwelingen $\mathrm{KG}$, et al. Impact of collateralisation to a concomitant chronic total occlusion in patients with STelevation myocardial infarction: a subanalysis of the EXPLORE randomised controlled trial. Open Heart 2018;5:e000810. doi:10.1136/ openhrt-2018-000810

Received 20 February 2018 Revised 9 April 2018 Accepted 24 April 2018
Check for updates

For numbered affiliations see end of article.

Correspondence to Dr Ivo $\mathrm{M}$ van Dongen; i.m. vandongen@amc.uva.nl

\section{ABSTRACT}

Objective The impact on cardiac function of collaterals towards a concomitant chronic total coronary occlusion (CTO) in patients with ST-elevation myocardial infarction (STEMI) has not been investigated yet. Therefore, we have evaluated the impact of well-developed collaterals compared with poorly developed collaterals to a concomitant CTO in STEMI.

Methods and results In the EXPLORE trial, patients with STEMI and a concomitant CTO were randomised to either CTO percutaneous coronary intervention (PCI) or no-CTO $\mathrm{PCl}$. Collateral grades were scored angiographically using the Rentrop grade classification. Left ventricular ejection fraction (LVEF) and left ventricular end-diastolic volume (LVEDV) at 4 months were measured using cardiac magnetic resonance imaging. Well-developed collaterals (Rentrop grades 2-3) to the CT0 were present in 162 (54\%) patients; these patients had a significantly higher LVEF at 4 months $(46.2 \pm 11.4 \%$ vs $42.1 \pm 12.7 \%, p=0.004)$ as well as a trend for a lower LVEDV $(208.2 \pm 55.7 \mathrm{~mL}$ vs $222.6 \pm 68.5 \mathrm{~mL}, p=0.054$ ) when compared with patients with poorly developed collaterals to the CTO. There was no significant difference in the total amount of scar in the two groups. Event rates were statistically comparable between patients with well-developed collaterals and poorly developed collaterals to the CTO at long-term follow-up.

Conclusions In patients with STEMI and a concomitant CTO, the presence of well-developed collaterals to a concomitant CTO is associated with a better LVEF at 4 months. However, this effect on LVEF did not translate into improvement in clinical outcome. Therefore, the presence of well-developed collaterals is important, but should not solely guide in the clinical decision-making process regarding any additional revascularisation of a concomitant CTO in patients with STEMI. Clinical trial registration NTR1108.

\section{Key questions}

What is already known about this subject?

- Previously, in patients treated for ST-elevation myocardial infarction (STEMI), the presence of well-developed collaterals to a concomitant coronary chronic total occlusion (СTO) has been associated with an improved survival.

What does this study add?

- This study shows that in patients treated for STEM the presence of well-developed collaterals is associated with a better left ventricular ejection fraction, but that the quality of collaterals to the CTO does not influence outcomes after revascularisation.

How might this impact on clinical practice?

- The results of this study suggest that in patients treated for STEMI the quality of collaterals to a concomitant CTO should not solely be leading when evaluating the therapeutic options with regard to the CTO.

\section{INTRODUCTION}

Chronic total coronary occlusions (CTOs) are frequently encountered in patients with coronary artery disease (CAD) (in 10-15\% of patients). ${ }^{1}$ Almost all of these patients demonstrate collateral filling of the CTO vessel to some degree. Even in patients with angiographic visible collaterals to the CTO less than $10 \%$ has a normal coronary flow reserve, which means that these collaterals cannot always protect the myocardium from hypoxia or intermittent ischaemia. ${ }^{2}$ In patients with ST-elevation myocardial infarction (STEMI), it has been known that good 
collateralisation of the infarct-related artery (IRA) is associated with improved clinical outcome. ${ }^{3}$ In addition, the presence of well-developed collaterals to a concomitant CTO also seems to be associated with improved survival compared with patient with poorly developed collaterals. ${ }^{4}$ However, little is known about the mechanisms involved.

The EXPLORE trial evaluated the impact of early percutaneous coronary intervention of a CTO (CTO PCI) versus no-CTO PCI in the first week after STEMI on left ventricular ejection fraction (LVEF) and left ventricular end-diastolic volume (LVEDV) on cardiac magnetic resonance imaging (CMR) at 4-month follow-up. The primary endpoint showed that there was no difference in LVEF and LVEDV between patients randomised to CTO PCI compared with patients randomised to no-CTO PCI.

We wanted to investigate the impact of well-developed collaterals versus poorly developed collaterals to a concomitant CTO in patients with STEMI on LVEF and LVEDV at 4-month CMR. We have also analysed whether collateralisation was associated with infarct size and we evaluated the effect of early CTO PCI on LVEF and LVEDV at 4 months and long-term clinical outcome in patients with well-developed collaterals and poorly developed collaterals.

\section{METHODS}

A total of 302 patients were enrolled in the EXPLORE trial. $^{5}$ After primary PCI (pPCI) for STEMI, all patients signed informed consent and baseline CMR was performed. Patients were then randomised to either CTO PCI within 7 days after pPCI or no early CTO PCI. At 4-month follow-up, patients underwent CMR or alternative imaging (eg, echocardiography and nuclear imaging) to assess the primary combined endpoint of LVEF and LVEDV. The primary endpoint of the study showed no difference between CTO PCI and no-CTO PCI on LVEF and LVEDV, and therefore we focused primarily on the presence of collaterals in this subanalysis. The study was conducted in accordance with the Declaration of Helsinki, and the study protocol was approved by institutional review boards of the participating institutes. More details of the trial design can be found in the design paper $^{6}$ and the main paper ${ }^{5}$ of the trial. In this substudy, we evaluated the quality of collaterals to the CTO in this STEMI population.

\section{Angiographic evaluation}

All baseline coronary angiograms, (non-)CTO PCI procedural characteristics, complications and success rates were adjudicated by two experienced interventional cardiologists ( $\mathrm{KGvH}$ and $\mathrm{PA})$. When they disagreed on collateral quality, all angios were reviewed in order to reach a consensus. The quality of collaterals was assessed at various time points:

1. On the pPCI angiogram before any intervention was performed.

2. Immediately after the pPCI procedure.
3. Before the CTO PCI procedure (within 7 days) in the CTO PCI randomised arm.

\section{Endpoint definitions}

The quality of the collaterals to the CTO was categorised into two groups based on the Rentrop collateral grading: Rentrop grade 0 for the absence of collaterals, grade 1 in the case of filling of side branches of the artery to be dilated via collateral channels without visualisation of the epicardial segment, grade 2 in case of partial filling of the epicardial segment and grade 3 for complete filling of the epicardial segment of the artery being dilated via collateral channels. Patients with Rentrop grades 2-3 collaterals formed the group of patients with well-developed collaterals (GOODCOLL) and all patients with Rentrop grades $0-1$ collaterals formed the group of patients with poorly developed collaterals (POORCOLL). ${ }^{7}$ All visible collaterals were assessed and the highest grade was used for categorisation.

A CTO was defined as antegrade thrombolysis in myocardial infarction $0-1$ flow over the lesion with an assumed duration of $\geq 3$ months. SYNTAX scores were calculated by Cardialysis BV, Rotterdam, The Netherlands, using the predefined SYNTAX score calculation definitions and website (http://www.syntaxscore.com/ calculator/syntaxscore/frameset.htm) ${ }^{89}$

Long-term follow-up (median 3.9 (IQR 2.9) years) has been presented previously. ${ }^{10}$ Follow-up was collected via patient-telephone contact. All events were adjudicated by an independent critical event committee. Major adverse cardiac events (MACE) were defined as the composite of cardiac death, myocardial infarction (MI) and coronary artery bypass grafting.

\section{Statistical analysis}

Continuous variables are depicted as mean $( \pm \mathrm{SD})$ or median (IQR), and comparisons between groups were made using independent t-test or non-parametric tests, respectively. In the case of comparing the delta change between baseline and 4-month imaging, the paired samples t-test was used. Categorical variables are depicted as frequencies (percentage of total), and comparisons between groups were made with the Fisher's exact test or $\mathrm{X}^{2}$ test when applicable. All-cause death event rates within groups are depicted with Kaplan-Meier curves and were compared using the log-rank test. Multivariable linear regression was used to evaluate the effect of baseline patient and angiographic characteristics on LVEF and LVEDV, and multivariable logistic regression was used to assess the effect of baseline patient and angiographic characteristics on the existence of well-developed collaterals. Appropriate variables were included using univariable linear and logistic regressions and after backward stepwise selection excluding variables with a $p$ value $>0.10$. The linearity assumption was checked visually and after confirmation that all Variance Inflation Factor (VIF) values were below 10 . 
Overall, a $\mathrm{p}$ value of $<0.05$ was considered statistically significant. The statistical analyses were carried out and figures were created with SPSS (V.24, IBM, Armonk, New York, USA) and Excel (V.14, Microsoft, Redmond, Washington, USA), respectively.

\section{RESULTS}

\section{Baseline patient and angiographic characteristics}

Of the 302 patients enrolled in EXPLORE, a total of 148 were randomised to the invasive arm and 154 to the conservative arm. One patient in the invasive treatment group refused the CTO PCI procedure, resulting in a total 147 patients who underwent CTO PCI at a median duration of 5 days after the pPCI. ${ }^{5}$

Table 1 shows all baseline characteristics in the overall population and in the GOODCOLL and POORCOLL groups. In all 302 patients, 162 patients (54\%) had GOODCOLL to the CTO vessel and 140 patients (46\%) had POORCOLL on the angiogram before pPCI. Patients in the GOODCOLL group had less frequently diabetes mellitus and hypertension compared with the POORCOLL group $(11 \%$ vs $21 \%$ and $36 \%$ vs $50 \%$, respectively). In half of patients in the GOODCOLL group, the IRA and CTO were located in the left anterior descending (LAD) and right coronary (RCA) arteries, respectively. Patients in the GOODCOLL group had less severe CAD, reflected by a lower SYNTAX score compared with the POORCOLL group. Also, baseline LVEF was significantly higher in patients with GOODCOLL compared with the baseline LVEF of patients with POORCOLL to the CTO $(42.9 \pm 10.9 \%$ vs $39.1 \pm 12.6 \%)$ (table 1).

Overall, in 80 patients (27\%) any collaterals to the IRA were observed. Of these, seven patients (9\%) had well-developed collaterals to the IRA. There was no difference in quality of collaterals to the IRA between patients with GOODCOLL and POORCOLL to the concomitant CTO (table 1).

Corrected for important patient and angiographic characteristics, both the SYNTAX score pre-pPCI and the presence of hypertension are independent negative predictors of GOODCOLL (table 2). The availability of GOODCOLL to the CTO is not an independent predictor for better LVEF nor LVEDV at 4-month follow-up (table 3A, B).

\section{Collaterals, left ventricular function and scar}

In total, 281 patients underwent CMR or alternative imaging at 4 months. Of these, 151 patients $(54 \%)$ were in the GOODCOLL group and 130 in the POORCOLL group. The mean LVEF in the GOODCOLL group was $46.3 \pm 11.5 \%$ at 4 -month follow-up and in the POORCOLL group $42.1 \pm 12.6 \% \quad(\mathrm{p}=0.004)$. The mean LVEDV at 4 months was $208 \pm 56 \mathrm{~mL}$ in the GOODCOLL group and $223 \pm 69 \mathrm{~mL}$ in the POORCOLL group $(\mathrm{p}=0.054)$. No difference in the amount of scar tissue was seen between both collateral groups (figure 1).
In figure 2, the LVEF and LVEDV at 4-month follow-up are depicted per highest Rentrop grade to the CTO lesion, showing that in patients without any collaterals to the CTO $(n=10)$, LVEF is lowest and LVEDV is highest compared with patients with collaterals of any Rentrop (1, 2 or 3$)$ grade.

In 180 patients, CMR was available at baseline in addition to the CMR at 4-month follow-up. ${ }^{11}$ In this group, 101 patients $(56 \%)$ had GOODCOLL to the CTO and 79 patients had POORCOLL. In the GOODCOLL group, the mean LVEF had increased significantly from $43.1 \pm 11.1 \%$ to $47.1 \pm 11.1 \%$ (mean delta $4.03 \pm 7.84 \%$, $\mathrm{p}<0.001)$ and in the POORCOLL group the LVEF also increased significantly from $38.9 \pm 12.6 \%$ at baseline to $43.3 \pm 12.1 \%$ at 4 -month follow-up (mean delta $4.42 \pm 8.61 \%, \mathrm{p}<0.001)$.

The change in mean LVEDV was not different between the GOODCOLL group $(208 \pm 52 \mathrm{~mL}$ at baseline, $211 \pm 48 \mathrm{~mL}$ at 4 months; mean delta $3.38 \pm 28.9 \mathrm{~mL}$, $\mathrm{p}=0.243)$ and the POORCOLL group $(212 \pm 57 \mathrm{~mL}$ at baseline, $218 \pm 61 \mathrm{~mL}$ at 4 months; mean delta $5.06 \pm 28.8 \mathrm{~mL}$, $\mathrm{p}=0.122$ ).

\section{Invasive treatment arm versus conservative arm}

In patients undergoing CTO PCI, 80 (54\%) had GOODCOLL to the CTO vessel and $67(46 \%)$ had POORCOLL. At 4-month follow-up, there was no difference in LVEF nor LVEDV between patients undergoing CTO PCI versus patients not undergoing CTO PCI in either the GOODCOLL or the POORCOLL group (online supplementary figure 1 , table 4 ).

\section{Collaterals and long-term outcomes}

MACE rates were comparable between patients with GOODCOLL and POORCOLL ( $8.0 \%$ vs $10.0 \%$, $\mathrm{p}=0.598$ ) at long-term follow-up. A trend towards more MIs can be appreciated in the POORCOLL group (table 5). All-cause mortality was statistically comparable between the two groups, with a numerically lower event rate in the GOODCOLL group versus the POORCOLL group ( $4.3 \%$ vs $7.1 \%, p=0.276$ ) on long-term follow-up (online supplementary figure 2 ).

Looking at the highest Rentrop grade for collaterals to the $\mathrm{CTO}$ as assessed during the pPCI procedure, patients with Rentrop grade 0 collaterals to the CTO appear to have significantly higher MACE and all-cause mortality rates compared with patients with higher Rentrop grades (online supplementary figure 3 ).

\section{DISCUSSION}

In the EXPLORE trial, patients with STEMI with well-developed collaterals to a concomitant CTO have a better LVEF and show a trend towards a better LVEDV on CMR at 4-month follow-up, compared with patients with poorly developed collaterals to the CTO. This difference at follow-up appears to be mainly driven by a difference in ejection fraction at baseline, as shown in the subgroup of patients with serial imaging. There was no treatment 
Open Heart

Table 1 Patient and angiographic baseline characteristics

\begin{tabular}{|c|c|c|c|c|}
\hline & All patients $(n=302)$ & GOODCOLL $(n=162)$ & POORCOLL $(n=140)$ & $P$ values \\
\hline Age & $60 \pm 10$ & $59 \pm 10$ & $61 \pm 10$ & 0.141 \\
\hline Male & $257(85 \%)$ & $138(85 \%)$ & $119(85 \%)$ & 1.000 \\
\hline Diabetes mellitus & $47(16 \%)$ & $17(11 \%)$ & $30(21 \%)$ & 0.011 \\
\hline Hypertension & $128(42 \%)$ & $58(36 \%)$ & $70(50 \%)$ & 0.014 \\
\hline Hyperlipidaemia & $103(34 \%)$ & $57(35 \%)$ & $46(33 \%)$ & 0.716 \\
\hline Current smoker & $153(51 \%)$ & $79(49 \%)$ & $74(53 \%)$ & 0.770 \\
\hline Previous Ml & $43(14 \%)$ & $25(15 \%)$ & $18(13 \%)$ & 0.621 \\
\hline Aspirin & $125(41 \%)$ & $66(41 \%)$ & $59(42 \%)$ & 0.331 \\
\hline Clopidogrel & $43(14 \%)$ & $25(\%)$ & $18(\%)$ & 0.383 \\
\hline Prasugrel & $4(1 \%)$ & $1(1 \%)$ & $3(2 \%)$ & 0.268 \\
\hline Ticagrelor & $29(10 \%)$ & $11(7 \%)$ & $18(13 \%)$ & 0.131 \\
\hline Coumarine & $1(0 \%)$ & $0(0 \%)$ & $1(1 \%)$ & 0.145 \\
\hline Beta-blocker & $63(21 \%)$ & $30(\%)$ & $33(\%)$ & 0.190 \\
\hline ACE inhibitor & $38(13 \%)$ & $17(10 \%)$ & $21(15 \%)$ & 0.163 \\
\hline Calcium-antagonist & $37(12 \%)$ & $16(\%)$ & $21(\%)$ & 0.135 \\
\hline ATII blocker & $31(10 \%)$ & $14(9 \%)$ & $17(12 \%)$ & 0.186 \\
\hline Infarct-related artery (IRA) & & & & $<0.001$ \\
\hline $\mathrm{RCA}$ & $93(31 \%)$ & $34(21 \%)$ & $59(42 \%)$ & \\
\hline LCX & $73(24 \%)$ & $43(27 \%)$ & $30(21 \%)$ & \\
\hline LAD & $136(45 \%)$ & $85(53 \%)$ & $51(36 \%)$ & \\
\hline TIMI culprit pre-PCI & & & & 0.274 \\
\hline $0-1$ & 199 (66\%) & $102(63 \%)$ & 97 (69\%) & \\
\hline $2-3$ & $103(34 \%)$ & $60(37 \%)$ & $43(31 \%)$ & \\
\hline Collaterals to IRA & $80(27 \%)$ & $40(25 \%)$ & $40(29 \%)$ & 0.431 \\
\hline GOODCOLL to IRA & $7(2 \%)$ & $3(2 \%)$ & $4(3 \%)$ & 1.000 \\
\hline CT0 artery & & & & $<0.001$ \\
\hline $\mathrm{RCA}$ & $142(47 \%)$ & $94(58 \%)$ & $48(34 \%)$ & \\
\hline LCX & $85(28 \%)$ & $41(25 \%)$ & $44(31 \%)$ & \\
\hline LAD & 75 (25\%) & 27 (17\%) & $48(34 \%)$ & \\
\hline \multicolumn{5}{|l|}{ TIMI CTO } \\
\hline $0-1$ & $300(99 \%)$ & $161(99 \%)$ & $139(99 \%)$ & \\
\hline 2 & $2(1 \%)$ & $1(1 \%)$ & $1(1 \%)$ & 1.000 \\
\hline Collateral grade to CTO & & & & - \\
\hline 0 & $10(3 \%)$ & - & $10(7 \%)$ & \\
\hline 1 & $130(43 \%)$ & - & $130(93 \%)$ & \\
\hline 2 & $143(47 \%)$ & $143(88 \%)$ & - & \\
\hline 3 & $19(6 \%)$ & $19(12 \%)$ & - & \\
\hline J-CTO score total & $2.2 \pm 1.1$ & $2.2 \pm 1.1$ & $2.2 \pm 1.2$ & 0.813 \\
\hline SYNTAX score & $29.2 \pm 9.2$ & $28.0 \pm 8.7$ & $30.6 \pm 9.5$ & 0.016 \\
\hline $\begin{array}{l}\operatorname{LVEF}(\%) \\
\operatorname{LVEDV}(\mathrm{mL})\end{array}$ & $\begin{array}{l}41.2 \pm 11.8 \\
211 \pm 53\end{array}$ & $\begin{array}{l}42.9 \pm 10.9 \\
209.0 \pm 51.8\end{array}$ & $\begin{array}{l}39.1 \pm 12.6 \\
213.2 \pm 55.2\end{array}$ & $\begin{array}{l}0.023 \\
0.577\end{array}$ \\
\hline \multicolumn{5}{|l|}{ Scar $(g)(n=149)$} \\
\hline Total & $8.5(4.7-15.1)$ & $7.3(4.2-14.6)$ & $8.9(5.4-17.2)$ & 0.212 \\
\hline Culprit area & $7.1(3.7-13.9)$ & $6.1(3.4-13.2)$ & $7.8(4.1-15.3)$ & 0.175 \\
\hline СT0 area & $0.7(0.1-1.6)$ & $0.7(0.2-1.5)$ & $0.8(0.1-1.8)$ & 0.982 \\
\hline
\end{tabular}


Table 1 Continued

\begin{tabular}{|c|c|c|c|c|}
\hline & All patients $(n=302)$ & GOODCOLL $(n=162)$ & POORCOLL $(n=140)$ & $P$ values \\
\hline \multicolumn{5}{|l|}{ Enzymatic infarct size } \\
\hline Peak CK-MB post pPCI & 124.5 (41.2-269.5) & $124.5(34.3-300.0)$ & $120.8(51.8-261.5)$ & 0.640 \\
\hline Peak troponin T post pPCl & $2.8(0.8-6.0)$ & $2.5(0.8-5.8)$ & $3.1(0.8-6.4)$ & 0.432 \\
\hline
\end{tabular}

ATII, Angiotensin-II receptor; CK-MB, creatinin kinase-myocardial band; CTO, coronary chronictotal occlusion; GOODCOLL, well-developed collaterals to the CTO; J-CTO, Japan chronic total occlusion; LAD, leftanterior descending artery; LCX, left circumflex artery; LVEDV, left ventricular end-diastolic volume; LVEF, left ventricular ejection fraction; MI, myocardial infarction; PCI, percutaneous coronary intervention; POORCOLL, poorly developed collaterals to the CTO; pPCI, primary PCI; $\mathrm{RCA}$, rightcoronary artery; TIMI, thrombolysis in myocardial infarction.

effect of CTO PCI within 7 days post STEMI, not in the GOODCOLL nor in the POORCOLL group. Interestingly, the quality of collaterals to the concomitant CTO in this STEMI population is not associated with final scar formation at 4-month follow-up.

\section{LVEF and the influence of collaterals}

At 4-month imaging, the ejection fraction is higher in patients with GOODCOLL compared with patients with POORCOLL, which seems to be driven by a higher ejection fraction at baseline in this group. This observed beneficial effect of GOODCOLL could be due to a better perfusion of the infarct border zone, especially when the infarct area and CTO area are overlapping. ${ }^{4}$ Hypothetically, better collaterals keep more myocardium viable, resulting in a better myocardial functionality that sustains on follow-up. ${ }^{4}$

In the CMR substudy of the EXPLORE trial, we did find more improvement in recovery of segmental wall thickening in dysfunctional segments in the CTO area on cardiac MRI in the patients with GOODCOLL compared with the patients with POORCOLL. ${ }^{11}$ Previous studies show conflicting results on the impact of collaterals on ejection fraction, but numbers were small. ${ }^{12} 13$ In our larger population, the difference in ejection fraction between patients with GOODCOLL and POORCOLL was large.
Also, patients without collaterals to their CTO (Rentrop grade 0 collaterals) had a worse ejection fraction and a worse prognosis compared with patients with any grade of collaterals (Rentrop grades 1-3).

\section{Scar size and collateralisation}

GOODCOLL have been suggested to ameliorate myocardial viability, leading to an improved myocardial functionality. ${ }^{4}$ However, this is not strongly supported by our data when looking at the subset of patients with scar-scoring on imaging: there is no statistically significant difference between patients with GOODCOLL and POORCOLL regarding the amount of total scar, nor regarding culprit area and CTO area scar. At baseline and 4-month imaging, the amount of scar remains small and statistically equal in both collateral groups. Interestingly, Werner et al have previously shown that there is no association between good collaterals and myocardial viability, ${ }^{14}$ which is thus supported by our data. Hypothetically, in the POORCOLL group, a larger amount of myocardium could remain viable but in hibernation as a protection mechanism from hypoxia, resulting in low scar formation and a lower functionality at baseline and follow-up.

\section{Collateral quality, MACE and all-cause mortality}

We found no clear difference in MACE event rates and survival, in contrast to our earlier registry data, ${ }^{4}$ between

Table 2 Influence of patient and angiographic characteristics on the development of GOODCOLL

\begin{tabular}{|c|c|c|c|c|c|c|}
\hline \multirow[b]{2}{*}{ Characteristic } & \multicolumn{3}{|c|}{ Univariable } & \multicolumn{3}{|c|}{ Multivariable } \\
\hline & OR & $95 \% \mathrm{Cl}$ & $P$ values & OR & $95 \% \mathrm{Cl}$ & $P$ values \\
\hline Age & 0.984 & 0.962 to 1.006 & 0.141 & - & - & - \\
\hline Male gender & 1.015 & 0.538 to 1.915 & 0.964 & - & - & - \\
\hline Hypertension & 0.558 & 0.352 to 0.885 & 0.013 & 0.583 & 0.366 to 0.929 & 0.023 \\
\hline Hypercholesterolaemia & 1.109 & 0.688 to 1.789 & 0.670 & - & - & - \\
\hline Diabetes mellitus & 0.430 & 0.226 to 0.819 & 0.010 & 0.620 & 0.266 to 1.442 & 0.267 \\
\hline SYNTAX score pre-pPCI & 0.970 & 0.945 to 0.995 & 0.017 & 0.972 & 0.947 to 0.997 & 0.031 \\
\hline LVEF at baseline & 1.030 & 1.005 to 1.055 & 0.019 & 1.020 & 0.994 to 1.046 & 0.127 \\
\hline
\end{tabular}

CTO, coronary chronictotal occlusion; GOODCOLL, well-developed collaterals to the CTO; LAD, left anterior descending artery; LCX, left circumflex artery; LVEDV, left ventricular end-diastolic volume; LVEF, leftventricular ejection fraction; MI, myocardial infarction; PCI, percutaneous coronary intervention; POORCOLL, poorly developed collaterals to the CTO; $\mathrm{PPCl}$, primary PCI; RCA, rightcoronary artery;

TIMI, thrombolysis in myocardial infarction. 
Table 3 Influence of patient and angiographic characteristics

\begin{tabular}{|c|c|c|c|c|c|c|}
\hline \multirow[b]{2}{*}{ Characteristic } & \multicolumn{3}{|c|}{ Univariable } & \multicolumn{3}{|c|}{ Multivariable } \\
\hline & $\beta$ & SE & $P$ values & $\beta$ & SE & $P$ values \\
\hline \multicolumn{7}{|l|}{ A-LVEF } \\
\hline Age & -0.088 & 0.072 & 0.226 & - & & \\
\hline Male gender & -0.393 & 2.039 & 0.847 & - & & \\
\hline Hypertension & -2.475 & 1.459 & 0.091 & 0.296 & 1.298 & 0.820 \\
\hline Hypercholesterolaemia & -2.086 & 1.528 & 0.173 & - & & \\
\hline Diabetes mellitus & -5.340 & 2.035 & 0.009 & 0.786 & 1.810 & 0.664 \\
\hline LVEF at baseline & 0.770 & 0.053 & $<0.001$ & 0.739 & 0.054 & $<0.001$ \\
\hline SYNTAX score pre-pPCl & -0.378 & 0.076 & $<0.001$ & -0.151 & 0.068 & 0.028 \\
\hline GOODCOLL to CTO & 4.187 & 1.437 & 0.004 & 0.578 & 1.294 & 0.655 \\
\hline \multicolumn{7}{|l|}{ B-LVEDV } \\
\hline Age & -0.364 & 0.370 & 0.327 & - & & \\
\hline Male gender & 37.365 & 10.185 & $<0.001$ & 8.650 & 8.331 & 0.301 \\
\hline Hypertension & 3.832 & 7.498 & 0.610 & - & & \\
\hline Hypercholesterolaemia & -5.495 & 7.833 & 0.484 & - & & \\
\hline Diabetes mellitus & 18.226 & 10.476 & 0.083 & 8.407 & 7.634 & 0.272 \\
\hline LVEDV at baseline & 0.889 & 0.051 & $<0.001$ & 0.870 & 0.051 & $<0.001$ \\
\hline SYNTAX score pre-pPCl & 1.839 & 0.393 & $<0.001$ & 0.695 & 0.291 & 0.018 \\
\hline GOODCOLL to CTO & -14.346 & 7.408 & 0.054 & -2.134 & 5.624 & 0.705 \\
\hline
\end{tabular}

Univariable and multivariable linear regression analyses investigating the effect of baseline characteristics on the left ventricular ejection fraction (LVEF (\%)), left ventricular end-diastolic volume (LVEDV $(\mathrm{mL})$ ) at 4-month follow-up. CTO, coronary chronic total occlusion; GOODCOLL, well-developed collaterals to the CTO; $\mathrm{PPCl}$, primary percutaneous coronary intervention.

patients with GOODCOLL and POORCOLL. This could be due to the absence of patients with cardiogenic shock in EXPLORE, who seem to benefit most from GOODCOLL, ${ }^{4}$ as well as due to the fact that EXPLORE was not powered for clinical events and the event rates are low overall.

\section{Clinical characteristics}

The clinical characteristics of patients in the GOODCOLL and POORCOLL groups were quite similar. However, patients with POORCOLL had more frequently diabetes mellitus and hypertension: baseline patient characteristics that have been associated with the development of a CTO. ${ }^{1}$ In our EXPLORE population, hypertension and the SYNTAX score pre-p PCI were independently associated (in a negative manner) with GOODCOLL. Other studies have found that female gender, diabetes mellitus and previous MI were more frequently seen in patients with POORCOLL, ${ }^{1516}$ while in another study hypertension was seen more frequently in patients with Rentrop grade 3 collaterals. ${ }^{12}$ Therefore, it appears difficult to attribute the quality of collaterals to the presence or absence of certain patient characteristics, although diabetes mellitus has been associated with a decrease in the quality of the cardiac microvasculature leading to decreased contractility and increased stiffness of the myocardium. ${ }^{17}$ Additionally, we have observed differences in angiographic characteristics: in patients with GOODCOLL, the IRA is significantly more frequently located in the LAD artery and the CTO lesion is more frequently located in the RCA, compared with patients with POORCOLL.

Another interesting finding is that we found a relatively low number of collaterals to the IRA and almost no GOODCOLL to this IRA. In recent STEMI studies, the occurrence of GOODCOLL to the IRA varied strongly (3\% and 22\%). ${ }^{34}$ This could be due to at least two reasons: the absence of collaterals to the IRA that would normally originate from the CTO vessel and/or the absence of cardiogenic shock in the aforementioned STEMI studies, which reduces the visibility of collaterals. ${ }^{4}$ However, in EXPLORE patients with cardiogenic shock were excluded, so very low systolic and high end-diastolic pressures are not the cause of the few collaterals to the IRA in our population. This suggests that the presence of a CTO lesion would be the most important cause for the few collaterals to the IRA vessel in these patients.

\section{Coronary chronic total occlusion percutaneous coronary intervention versus no-Coronary chronic total occlusion percutaneous coronary intervention}

We found no clear effect of CTO PCI in either of the collateral groups. This is in contrast to a study by Choi et al, who found a significant increase in ejection fraction from pre-CTO PCI to follow-up at 1.7 years in a population of patients with stable CAD and well-developed (Rentrop grades 2-3) collaterals to a CTO, compared with patients with well-developed collaterals receiving optical medical 

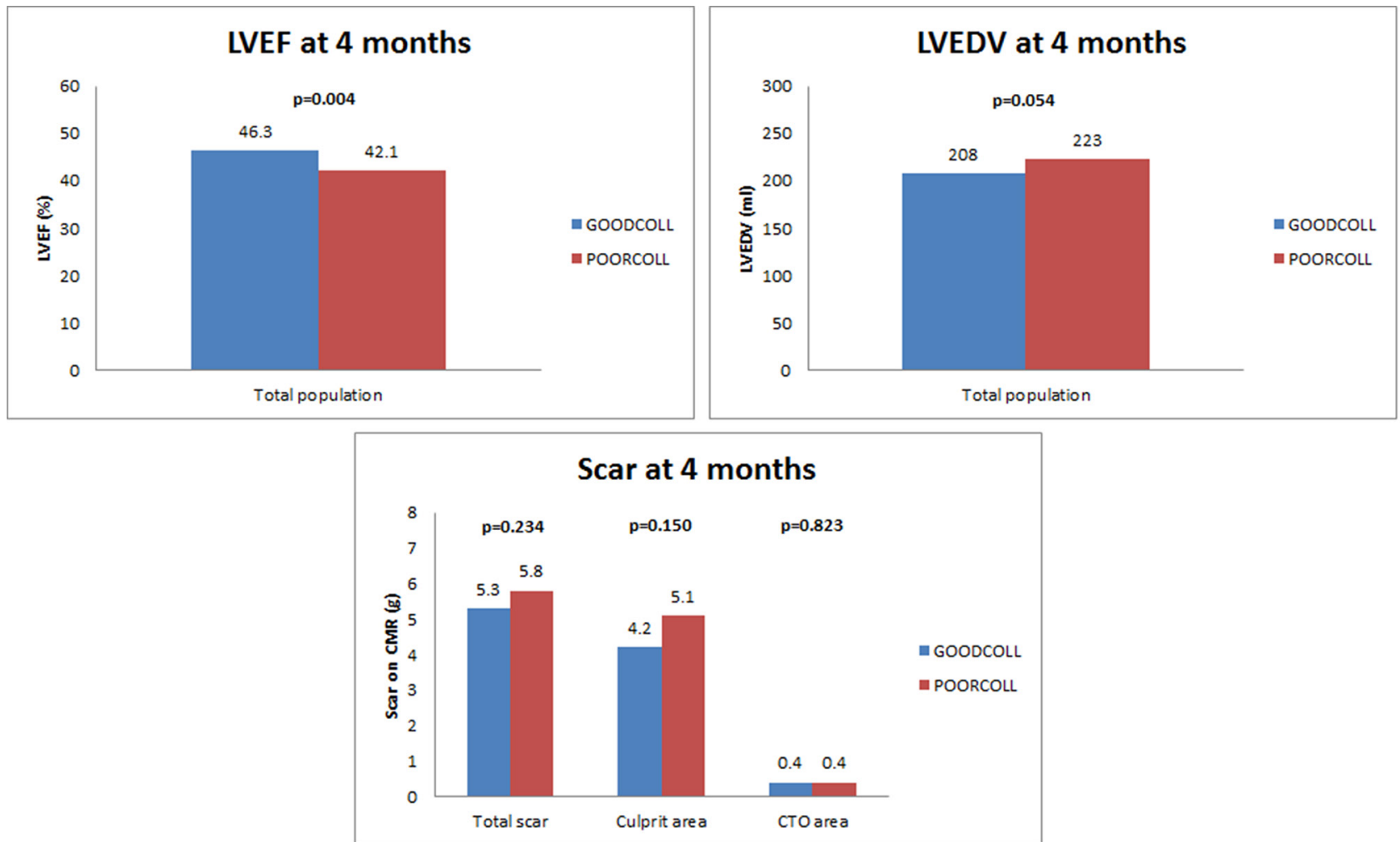

Figure 1 The effect of GOODCOLL versus POORCOLL on LVEF, LVEDV and scar at 4 months. LVEF, LVEDV and scar at 4- month follow-up in the total population, comparing GOODCOLL (well-developed collaterals to the CTO) and POORCOLL (poorly developed collaterals to the CTO) (first two upper panels) and the total amount of scar, amount of scar in the culprit vessel area and CTO vessel area in patients with GOODCOLL and POORCOLL (lower panel). CMR, cardiac magnetic resonance imaging; CTO, coronary chronic total occlusion; LVEDV, left ventricular end-diastolic volume (mL); LVEF, left ventricular ejection fraction (\%).

treatment. ${ }^{18}$ In addition, in the Bleeding Complications in a Multicenter Registry of Patients Discharged With Diagnosis of Acute Coronary Syndrome (BleeMACS) registry, patients with STEMI treated with complete revascularisation experienced lower mortality rates, compared with patients with STEMI receiving incomplete revascularisation. ${ }^{19}$ Our study population is too small to do similar analyses, but overall we did not find a clear difference between collateral groups and CTO PCI versus no-CTO PCI. Collateralisation status, thus, does not clearly influence the outcomes of revascularisation.

\section{LIMITATIONS}

This is a subanalysis of a larger randomised controlled trial. Also, the EXPLORE trial was not powered for clinical follow-up and the number of clinical events is relatively small. Angiographic assessment of collaterals is limited, ${ }^{20}$ so the presence and amount of any other smaller collateral vessels have not been taken into account in this analysis. Also, we did not have any data on covariables that could influence ventricular remodelling (such as mitral regurgitation).
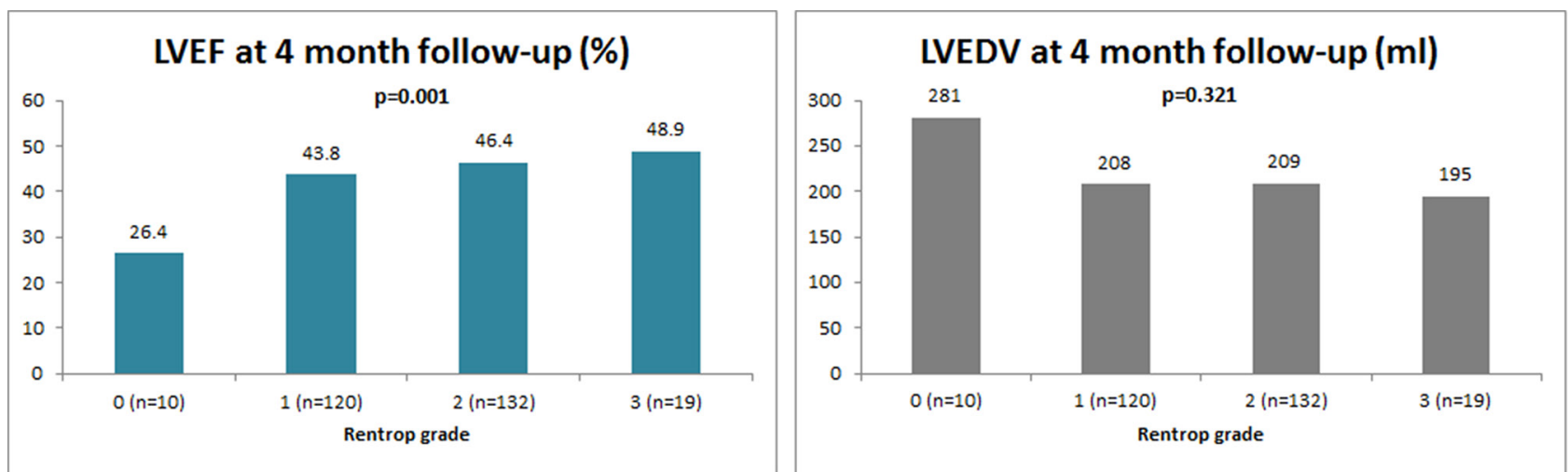

Figure 2 The impact of Rentrop grade collaterals to the coronary chronic total occlusion on left ventricular ejection fraction (LVEF) and left ventricular end-diastolic volume (LVEDV). 
Table 4 Comparison of the influence of CTO PCI versus no-CTO PCI on LVEF and LVEDV at 4 months in the GOODCOLL and POORCOLL groups

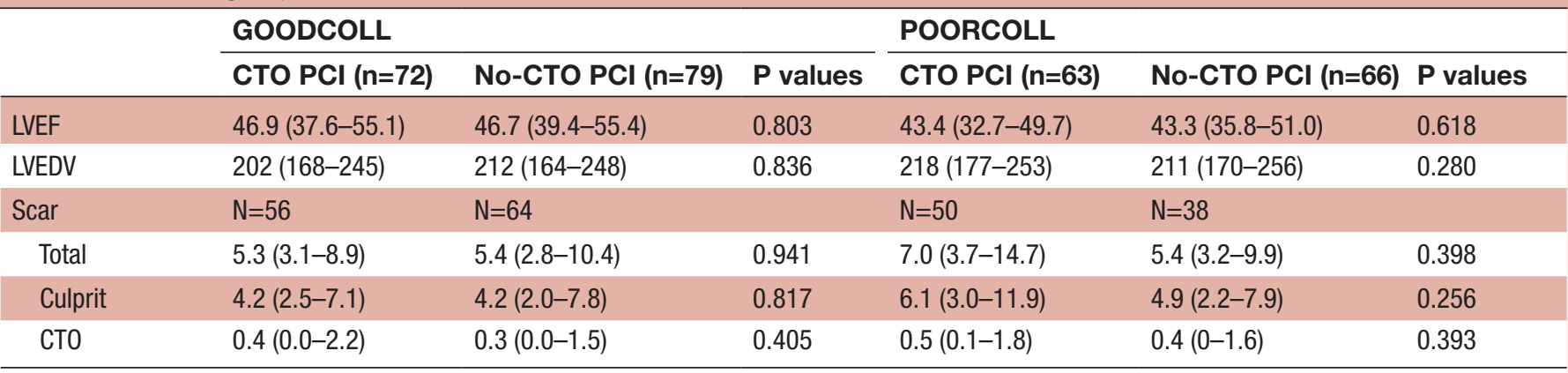

CTO, coronary chronic total occlusion; GOODCOLL, well-developed collaterals to the CTO; LVEDV, left ventricular end-diastolic volume (mL); LVEF, left ventricular ejection fraction (\%); PCI, percutaneouscoronary intervention; POORCOLL, poorlydeveloped collaterals to the CTO.

\begin{tabular}{cccc}
\hline Table 5 & Event rates at long-term follow-up & \\
\hline & $\begin{array}{l}\text { GOODCOLL } \\
\text { (n=162) }\end{array}$ & $\begin{array}{l}\text { POORCOLL } \\
\text { (n=140) }\end{array}$ & P values \\
\hline All-cause death & $8(5 \%)$ & $13(9 \%)$ & 0.174 \\
Composite MACE & $16(10 \%)$ & $20(14 \%)$ & 0.286 \\
Cardiac death & $4(3 \%)$ & $5(4 \%)$ & 0.738 \\
All MI & $9(6 \%)$ & $16(11 \%)$ & 0.092 \\
CABG & $4(3 \%)$ & $4(3 \%)$ & 1.000 \\
\hline
\end{tabular}

CABG, coronary artery bypass grafting; CTO, coronary chronic total occlusion; GOODCOLL, well-developed collaterals to the CTO; MACE, major adverse cardiac events; MI, myocardial infarction; POORCOLL, poorly developed collaterals to the CTO.

\section{CONCLUSION}

In this subanalysis of the EXPLORE trial, we show that in patients with STEMI with a concomitant CTO, the presence of well-developed collaterals to the CTO is associated with a better LVEF at 4-month follow-up. Collateralisation to a CTO vessel was not associated with final scar size at 4-month follow-up. Also, collateral quality to the CTO was not clearly associated with MACE nor all-cause mortality rates. The presence of well-developed collaterals is thus important, but should not be leading in the evaluation of the potential benefit of additional revascularisation of a concomitant CTO in patients with STEMI.

\section{Author affiliations}

${ }^{1}$ Academic Medical Center, University of Amsterdam, Amsterdam, The Netherlands ${ }^{2}$ Medisch Spectrum Twente, Enschede, The Netherlands

${ }^{3}$ St. Antonius Ziekenhuis, Nieuwegein, The Netherlands

${ }^{4}$ Sahlgrenska University Hospital, Gothenburg, Sweden

${ }^{5}$ North Estonia Medical Center, Tallinn, Estonia

${ }^{6}$ Haukeland University Hospital, Bergen, Norway

${ }^{7}$ Haga Teaching Hospital, The Hague, The Netherlands

${ }^{8}$ Onze Lieve Vrouwe Gasthuis, Amsterdam, The Netherlands

Acknowledgements The authors thank the investigators of the EXPLORE trial and the medical and nursing staff in the participating centres. The authors also thank all patients who participated in this trial.

Funding The EXPLORE trial is an investigator-initiated study and was funded by the Academic Medical Center (Amsterdam, The Netherlands) in combination with an unrestricted research grant from Abbott Vascular (Santa Clara, California, USA).
Competing interests Professor JPSH has received grants from Abbott Vascular during the conduct of the study and has received grants from BBraun, Abiomed and Biotronik outside the submitted work; Dr RJvanderS has received grants from Abbott Vascular, Biotronik and Biosensors and has received personal fees from Biotronik and Boston Scientific, has been a consultant for Biotronik and has received speaker fees from OrbusNeich, Boston Scientific and Asahi Intecc outside the submitted work; Dr TR has been a proctor for Boston Scientific and all other authors have reported that they have no relationships relevant to the contents of this paper to disclose.

Patient consent written consent was acquired for the trial

Ethics approval Academic Medical Center ethics committee/institutional review board.

Provenance and peer review Not commissioned; externally peer reviewed.

Open access This is an open access article distributed in accordance with the Creative Commons Attribution Non Commercial (CC BY-NC 4.0) license, which permits others to distribute, remix, adapt, build upon this work non-commercially, and license their derivative works on different terms, provided the original work is properly cited and the use is non-commercial. See: http://creativecommons.org/ licenses/by-nc/4.0/

(C) Author(s) (or their employer(s)) 2018. Re-use permitted under CC BY-NC. No commercial re-use. See rights and permissions. Published by BMJ.

\section{REFERENCES}

1. Hoebers LP, Claessen BE, Dangas GD, et al. Contemporary overview and clinical perspectives of chronic total occlusions. Nat Rev Cardiol 2014;11:458-69.

2. Werner GS, Surber R, Ferrari M, et al. The functional reserve of collaterals supplying long-term chronic total coronary occlusions in patients without prior myocardial infarction. Eur Heart $J$ 2006;27:2406-12.

3. Kim EK, Choi JH, Song YB, et al. A protective role of early collateral blood flow in patients with ST-segment elevation myocardial infarction. Am Heart J 2016;171:56-63.

4. Elias J, Hoebers LPC, van Dongen IM, et al. Impact of Collateral Circulation on Survival in ST-Segment Elevation Myocardial Infarction Patients Undergoing Primary Percutaneous Coronary Intervention With a Concomitant Chronic Total Occlusion. JACC Cardiovasc Interv 2017;10:906-14

5. Henriques JP, Hoebers LP, Råmunddal T, et al. Percutaneous Intervention for Concurrent Chronic Total Occlusions in Patients With STEMI: The EXPLORE Trial. J Am Coll Cardiol 2016;68:1622-32.

6. van der Schaaf RJ, Claessen BE, Hoebers LP, et al. Rationale and design of EXPLORE: a randomized, prospective, multicenter trial investigating the impact of recanalization of a chronic total occlusion on left ventricular function in patients after primary percutaneous coronary intervention for acute ST-elevation myocardial infarction. Trials 2010;11:89.

7. Rentrop KP, Cohen M, Blanke $\mathrm{H}$, et al. Changes in collateral channel filling immediately after controlled coronary artery occlusion by an angioplasty balloon in human subjects. J Am Coll Cardiol 1985;5:587-92. 
8. Magro M, Nauta S, Simsek C, et al. Value of the SYNTAX score in patients treated by primary percutaneous coronary intervention for acute ST-elevation myocardial infarction: The MI SYNTAXscore study. Am Heart J 2011;161:771-81.

9. Sianos G, Morel MA, Kappetein AP, et al. The SYNTAX Score: an angiographic tool grading the complexity of coronary artery disease. Eurolntervention 2005;1:219-27.

10. Elias J, van Dongen IM, Råmunddal T, et al. 2035Mid- and long-term outcome of the EXPLORE trial: investigating the impact of CTO PCl versus no-CTO PCI in STEMI patients with a concurrent CTO. Eur Heart J 2017;38:ehx502.2035-ehx502.

11. Elias J, van Dongen IM, Hoebers LP, et al. Improved recovery of regional left ventricular function after $\mathrm{PCl}$ of chronic total occlusion in STEMI patients: a cardiovascular magnetic resonance study of the randomized controlled EXPLORE trial. J Cardiovasc Magn Reson 2017;19:53.

12. Aboul-Enein F, Kar S, Hayes SW, et al. Influence of angiographic collateral circulation on myocardial perfusion in patients with chronic total occlusion of a single coronary artery and no prior myocardial infarction. J Nucl Med 2004;45:950-5.

13. Ajayi NO, Vanker EA, Satyapal KS. The role of coronary artery collaterals in the preservation of left ventricular function: a study to address a long-standing controversy. Cardiovasc J Afr 2017;28:81-5.
14. Werner GS, Surber R, Kuethe F, et al. Collaterals and the recovery of left ventricular function after recanalization of a chronic total coronary occlusion. Am Heart J 2005;149:129-37.

15. van der Hoeven NW, Teunissen PF, Werner GS, et al. Clinical parameters associated with collateral development in patients with chronic total coronary occlusion. Heart 2013;99:1100-5.

16. Yetkin E, Topal E, Erguzel N, et al. Diabetes mellitus and female gender are the strongest predictors of poor collateral vessel development in patients with severe coronary artery stenosis. Angiogenesis 2015;18:201-7.

17. Hinkel R, Howe A, Renner S, et al. Diabetes Mellitus-Induced Microvascular Destabilization in the Myocardium. J Am Coll Cardiol 2017;69:131-43.

18. Choi SY, Choi BG, Rha SW, et al. Percutaneous coronary intervention versus optimal medical therapy for chronic total coronary occlusion with well-developed collaterals. J Am Heart Assoc 2017;6:e006357.

19. Quadri G, D'Ascenzo F, Moretti C, et al. Complete or incomplete coronary revascularisation in patients with myocardial infarction and multivessel disease: a propensity score analysis from the "real-life" BleeMACS (Bleeding complications in a Multicenter registry of patients discharged with diagnosis of Acute Coronary Syndrome) registry. Eurolntervention 2017;13:407-14.

20. Traupe T, Gloekler S, de Marchi SF, et al. Assessment of the human coronary collateral circulation. Circulation 2010;122:1210-20. 